\title{
Laplacian, on the Arrowhead Curve
}

\author{
Claire David
}

\begin{abstract}
In terms of analysis on fractals, the Sierpiński gasket stands out as one of the most studied example. The underlying aim of those studies is to determine a differential operator equivalent to the classic Laplacian. The classically adopted approach is a bidimensional one, through a sequence of so-called prefractals, i.e. a sequence of graphs that converges towards the considered domain. The Laplacian is obtained through a weak formulation, by means of Dirichlet forms, built by induction on the prefractals.

It turns out that the gasket is also the image of a Peano curve, the so-called Arrowhead one, obtained by means of similarities from a starting point which is the unit line. This raises a question that appears of interest. Dirichlet forms solely depend on the topology of the domain, and not of its geometry. Which means that, if one aims at building a Laplacian on a fractal domain as the aforementioned curve, the topology of which is the same as, for instance, a line segment, one has to find a way of taking account its specific geometry.

Another difference due to the geometry, is encountered may one want to build a specific measure. For memory, the sub-cells of the Kigami and Strichartz approach are triangular and closed: the similarities at stake in the building of the Curve called for semi-closed trapezoids. As far as we know, and until now, such an approach is not a common one, and does not appear in such a context.

It intererestingly happens that the measure we choose corresponds, in a sense, to the natural counting measure on the curve. Also, it is in perfect accordance with the one used in the Kigami and Strichartz approach. In doing so, we make the comparison - and the link - between three different approaches, that enable one to obtain the Laplacian on the arrowhead curve: the natural method; the Kigami and Strichartz approach, using decimation; the Mosco approach.
\end{abstract}

Keywords: Laplacian, arrowhead curve, Sierpiński gasket

Ключові слова: лапласіан, крива-стрілка, килим Серпінського

DOI: http://dx.doi.org/10.15673/tmgc.v13i2.1746 


\begin{abstract}
Анотація. 3 точки зору аналізу килим Серпінського є одним 3 найбільш вивчених фракталів, причому більшість аналітичних досліджень на ньому присвячена визначенню диференціального оператора, який був би еквівалентним до класичного лапласіана. Класично прийнятий підхід полягає в тому, щоб задати килим Серпінського як межу послідовності так званих префракталів, тобто графіків деяких функцій. В такій ситуації, Лапласіан визначають як слабку межу (в термінах форм Діріхле) Лапласіанів на префракталах.

3 іншого боку, килим Серпінського є також границею кривої Пеано, так званої "стрілки”, отриманої за допомогою подібностей з одиничного відрізка. Ця крива вже не є самоподібною і має топологію, що суттєво відрізняється від топології килима Серпінського. Відмітимо, що форми Діріхле залежать виключно від топології області, а не від її геометрії. Тому для того, щоб визначити лапласіан на фрактальній множині, такій як "стрілка", потрібно враховувати її метричні властивості як підмножини площини. В даній роботі ми визначаємо Лапласіан на "стрілці" за допомогою побудови міри, яка гарно узгоджується з підходами Дж. Кігамі та Р. Стріхарца, а також порівнюємо побудову з методом У. Моско.
\end{abstract}

\title{
1. InTRODUCTION
}

In $[11,12]$, Jun Kigami has laid the foundations of what is now known as differential calculus on fractals, by allowing the construction of an operator of the same nature of the Laplacian, defined locally, on graphs having a fractal character. The Sierpiński gasket stands out of the best known example. It has, since then, been taken up, developed and popularized by Robert S. Strichartz [20, 21].

The Laplacian is obtained through a weak formulation, by means of Dirichlet forms, built by induction on a sequence of graphs that converges towards the considered domain. It is these Dirichlet forms that enable one to obtain energy forms on this domain.

It is interesting to note that the existing results mainly deal with nested fractals, generated by an iterated function system with at least two fixed points, connected, symmetric (a classical example is the Vicsek snowflake), or post-critically finite (p.c.f.) ones. Both categories have in common to be self-similar.

A step forward has been made by Uta Freiberg and Maria Rosaria Lancia [3], where the authors build an energy form on non-self similar closed fractal curves, by integrating the Lagrangian on these curves, following the works of Umberto Mosco [15].

Yet, things are not that simple, and interesting questions arise on the scene. One may wonder what happens when a fractal set can be obtained by two different processes, first, by means of an iterated function system, 
second, by means of a $L$-system where the same self-similarity no longer seems to hold, as it is the case of the Sierpiński gasket?

The Sierpiński gasket is, in fact, the limit of the so-called Sierpiński arrowhead curve (see [14, plate 141] and [16, page 346]). First, the curve has a completely different topology, second, contrary to the triangle, it can be thought to belong to the category of Moran fractals (we refer to $[2,9]$ ), since, at first sight (only), it is not self-similar as the gasket. Yet, in so far as the limit curve reaches each point of the gasket, and as explained by Benoît Mandelbrot, by "fusing together four appropriately neighboring tiles, one gets a tile increased in the ratio of 2", it appears legitimate to consider the limit curve as self-similar. There does not seem to be other references to the arrowhead curve in the literature, and none dealing with the building of a Laplacian on such a curve.

This brings us to a question that appears of interest. Dirichlet forms solely depend on the topology of the domain, and not of its geometry. Which means that, if one aims at building a Laplacian on a fractal domain as the aforementioned curve, the topology of which is the same as, for instance, a line segment, one has to find a way of taking account such a specific geometry.

Building a Laplacian on the arrowhead curve has thus led us to use methods that are not so standard. May one want to follow the work of J. Kigami and R. S. Strichartz, it requires a measure. J. Kigami and R. S. Strichartz choose to build measures that were more like the Riemann or Cauchy ones, rather than the Lebesgue one. To this purpose, they introduced, first, a regular probability measure, where each subcell of level $m \in \mathbb{N}^{\star}$, a triangular one, has measure $\frac{1}{3^{m}}$. This measure happened to coincide, up to a constant, to the Hausdorff measure of the triangle. It is worth noting that the considered integral is thus a two dimensional one.

The Sierpiński gasket can be considered as a curve: it is, as evoked in the above, the limit of the so-called Sierpiński arrowhead curve. The point is that, generally, the Hausdorff measure is not suited for integration along fractal curves, due to dissimilarities between its properties, and the one of integrals along curves, as it is well explained in the paper by Boris Kats [10]: for a fractal set $\mathcal{F}$ of the complex plane $\mathbb{C}$, the Hausdorff dimension of which is $\alpha \in\{1,2\}$, the related Hausdorff measure $m_{\alpha}$ is such that the function

$$
z \in \mathbb{C} \mapsto \frac{1}{2 \pi i} \int_{\mathcal{F}} \frac{u(\zeta)}{\zeta-z} d m_{\alpha}(\zeta)
$$

is continuous on the whole complex plane, whereas the integral

$$
\frac{1}{2 \pi i} \int_{\mathcal{F}} \frac{u(\zeta)}{\zeta-z} d \zeta
$$


has unit gaps on $\mathcal{F}$.

For whom aims at studying diffusion in such media, it appears as more appropriate to consider the integral over the whole area delimited by the curve, rather than the one along the curve.

To this purpose, we found it all the more natural to introduce a sequence of trapezes $\left(\mathcal{T}_{m j}\right)_{m \in \mathbb{N}}, 1 \leqslant j \leqslant 3^{m-1}$, delimitating a trapezoidal domain infinitely close to the arrowhead curve $\mathcal{S} G^{\mathcal{C}}, \mathcal{D}\left(\mathcal{S} G^{\mathcal{C}}\right)$. In the literature, one can already find approximating polygons, for instance in the case of the Peano curve, as introduced by W. Wunderlich [24]. Such a notion was then adopted by H. Sagan $[18,19]$.

It also seemed natural, in the sense that it enables one to take into account the trapezoidal replicated patterns that appear thanks to the curve, patterns, the measure of which plays the part of a pound. This joins the seminal work of J. Harrison et al. [7, 8, 6], in the spirit of the one by H. Withney [23], and the remark of U. Mosco [15], where the author suggests, in the case of the Sierpinski curve, to "fill each small simplex (...) not only with its edges, but with the whole portion of the limit curve which it encompasses."

Again, one encounters a difference due to the geometry, since the sub-cells of the Kigami and Strichartz approach are triangular and closed, whereas ours are semi-closed trapezoids. As far as we know, and until now, such an approach is not a common one, and does not appear in such a context.

It interestingly happens that the measure we choose corresponds, in a sense, to the natural counting measure on the curve. Also, it is in perfect accordance with the one used in the Kigami and Strichartz approach, as we will see it further. In doing so, we make the comparison - and the link between three different approaches, that enable one to obtain the Laplacian on the arrowhead curve: the natural method; the Kigami and Strichartz approach [13, 21], using decimation; the Mosco approach [15].

Thus, we aim at investigating the links between energy forms and geometry that can arise in such cases. Does one obtain the same Laplacian as for the triangle? The question appears as worth to be investigated.

\section{FrAmEWORK OF THE STUDY}

In the sequel, we place ourselves in the Euclidean plane of dimension 2, referred to a direct orthonormal frame. The usual Cartesian coordinates are $(x, y)$. We will denote by $\mathbb{N}$ the set of natural integers, and set:

$$
\mathbb{N}^{\star}=\mathbb{N} \backslash\{0\} .
$$

Definition 2.1. Given a strictly positive integer $k$, two integers $a$ and $b$ will be called congruent modulo $k$ if $a-b$ is divisible by $k$. The congruence 
modulo $k$ between $a$ and $b$ will be denoted:

$$
a \equiv b[k]
$$

Notation 2.2. We will denote by:

$$
D_{\mathcal{S} G^{\mathcal{C}}}=D_{\mathcal{S} G}=\frac{\ln 3}{\ln 2}
$$

the box dimension (equal to the Hausdorff dimension), of the Sierpiński arrowhead curve $\mathcal{S} G^{\mathcal{C}}$. For the sake of simplicity, we will from now on denote it by $D_{\mathcal{S} G}$.

Notation 2.3. Given a point $X \in \mathbb{R}^{2}$, we will denote by:

i) $\operatorname{Sim}_{X, \frac{1}{2}, \frac{\pi}{3}}$ the similarity of ratio $\frac{1}{2}$, the center of which is $X$ and the angle $\frac{\pi}{3}$

ii) $\operatorname{Sim}_{X, \frac{1}{2},-\frac{\pi}{3}}$ the similarity of ratio $\frac{1}{2}$, the center of which is $X$ and the angle $-\frac{\pi}{3}$.

Definition 2.4. Consider the following points of $\mathbb{R}^{2}$ :

$$
A=(0,0), \quad D=(1,0), \quad B=\operatorname{Sim}_{A, \frac{1}{2}, \frac{\pi}{3}}(D), \quad C=\mathcal{S i m}_{D, \frac{1}{2},-\frac{\pi}{3}}(A) .
$$

We will denote by $V_{1}$ the ordered set, of the points:

$$
\{A, B, C, D\} \text {. }
$$

The set of points $V_{1}$, where $A$ is linked to $B, B$ is linked to $C$, and where $C$ is linked to $D$, constitutes an oriented graph, that we will denote by $\mathcal{S} G_{1}^{C}$. The set $V_{1}$ is called the set of vertices of the graph $\mathcal{S} G_{1}^{\mathcal{C}}$.

Let us build by induction the sequence of points:

$$
\left(V_{m}\right)_{m \in \mathbb{N}^{\star}}=\left(X_{j}^{m}\right)_{1 \leqslant j \leqslant \mathcal{N}_{m}^{\mathcal{S}}, m \in \mathbb{N}^{\star}}, \quad \mathcal{N}_{m}^{\mathcal{S}} \in \mathbb{N}^{\star}
$$

such that:

$$
X_{1}^{1}=A, \quad X_{2}^{1}=B, \quad X_{3}^{1}=A, \quad X_{4}^{1}=D,
$$

and for any integers $m \geqslant 2,1 \leqslant j \leqslant \mathcal{N}_{m}^{\mathcal{S}}, k \in \mathbb{N}, \ell \in \mathbb{N}$ :

$$
\begin{aligned}
& X_{j+k}^{m}=X_{j}^{m-1}, \quad \text { if } k \equiv 0 \text { [3], } \\
& X_{j+k+\ell}^{m}=\mathcal{S i m}_{X_{j+\ell}^{m-1}, \frac{1}{2},(-1)^{m+j+\ell+k+1} \frac{\pi}{3}}\left(X_{j+\ell+1}^{m-1}\right) \text {, if } k \equiv 1[3] \text { and } \ell \in 2 \mathbb{N} \text {, } \\
& X_{j+k+\ell}^{m}=\mathcal{S i m}_{X_{j+\ell+1}^{m-1}, \frac{1}{2},(-1)^{m+j+\ell+k+1} \frac{\pi}{3}}\left(X_{j+\ell}^{m-1}\right), \quad \text { if } k \equiv 2[3] \text { and } \ell \in \mathbb{N} \backslash 2 \mathbb{N} \text {. }
\end{aligned}
$$

The set of points $V_{m}$, where two consecutive points are linked, is an oriented graph, which we will denote by $\mathcal{S} G_{m}^{\mathcal{C}}$. The set $V_{m}$ is called the set of vertices of the graph $\mathcal{S} G_{m}^{\mathcal{C}}$. 
Property 2.5. For any strictly positive integer $m$ :

$$
V_{m} \subset V_{m+1} \text {. }
$$

Property 2.6. If one denotes by $\left(\mathcal{S} G_{m}\right)_{m \in \mathbb{N}}$ the sequence of graphs that approximate the Sierpiński gasket $\mathcal{S} G$, then, for any strictly positive integer $m$ :

$$
\mathcal{S} G_{m}^{\mathcal{C}} \subsetneq \mathcal{S} G_{m}
$$

Definition 2.7. We will denote by $\mathcal{S} G^{\mathcal{C}}$ the limit, in the space of continuous functions from the interval to the plane:

$$
\mathcal{S} G^{\mathcal{C}}=\lim _{m \rightarrow+\infty} \mathcal{S} G_{m}^{\mathcal{C}}
$$

This limit will be called the Sierpiński arrowhead curve.

Property 2.8. Denote by $\mathcal{S} G$ the Sierpiński gasket. Then:

$$
\lim _{m \rightarrow+\infty} \mathcal{S} G_{m}^{\mathcal{C}}=\mathcal{S} G^{\mathcal{C}}=\mathcal{S} G
$$

Remark 2.9. The sequence of graphs $\left(\mathcal{S} G_{m}\right)_{m \in \mathbb{N}^{\star}}$ can also be seen as a Lindenmayer system ("L-system"), i.e. a set $(V, \omega, P)$, where $V$ denotes an alphabet (or, equivalently, the set of constant elements and rules, and variables), $\omega$, the initial state (also called "axiom"), and $P$, the production rules, which are to be applied, iteratively, to the initial state.

In the case of the Sierpiński arrowhead curve, if one denotes by:

i) F the rule: "Draw forward, on one unit length";

ii) + the rule: "Turn left, with an angle of $\frac{\pi}{3}$ ";

iii) - the rule: "Turn right, with an angle of $\frac{\pi}{3}$ "; then:

i) the variables can be denoted by $X$ and $Y$;

ii) the constants are $F,+,-$;

iii) the initial state is $X F$;

iv) the production rules are:

$$
X \rightarrow Y F+X F+Y, \quad Y \rightarrow X F-Y F-X .
$$

Notation 2.10. Given a point $X \in \mathbb{R}^{2}$, we will denote by $\operatorname{Sim}_{X, \frac{1}{2}, 0}$ the homothety of ratio $\frac{1}{2}$, the center of which is $X$.

Property 2.11 (Self-similarity properties of Sierpiński arrowhead curve). Denote by $E$ the point of $\mathbb{R}^{2}$ such that $A, D$ and $E$ are the consecutive vertices of a direct equilateral triangle. One may note that $A, D$ and $E$ are, also, the frontier vertices of the Sierpiński gasket $\mathcal{S} G$. The Sierpiński 


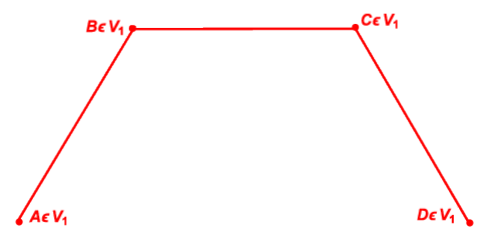

The graph $\mathcal{S} G_{1}^{\mathcal{C}}$

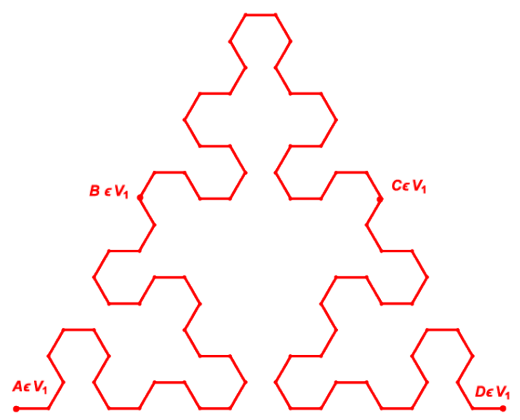

The graph $\mathcal{S} G_{4}^{\mathcal{C}}$

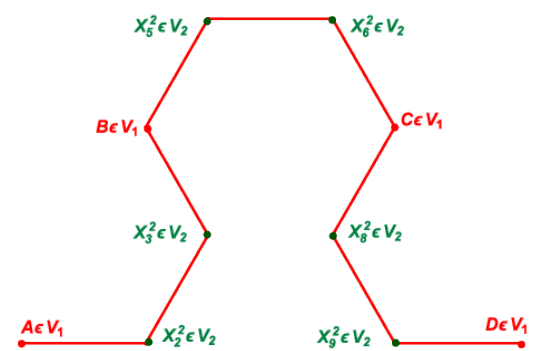

The graph $\mathcal{S} G_{2}^{\mathcal{C}}$

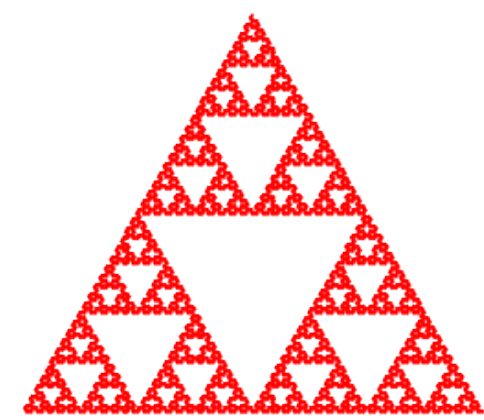

The graph $\mathcal{S} G_{7}^{\mathcal{C}}$

FiguRe 2.1.

arrowhead curve is self similar with the three homotheties:

$$
\mathcal{H}_{1}=\operatorname{Sim}_{A, \frac{1}{2}, 0}, \quad \mathcal{H}_{2}=\operatorname{Sim}_{D, \frac{1}{2}, 0}, \quad \mathcal{H}_{3}=\mathcal{S i m}_{E, \frac{1}{2}, 0} .
$$

Proof. The result comes from the self-similarity of the Sierpiński gasket with respect to those homotethies:

$$
\mathcal{S} G=\bigcup_{i=1}^{3} \mathcal{H}_{i}(\mathcal{S} G)
$$

Notation 2.12. For any strictly positive integer $m$, we will denote by $\mathcal{N}_{m}^{\mathcal{S}}$ the number of vertices of $\mathcal{S} G_{m}^{\mathcal{C}}$.

Property 2.13. The sequence $\left(\mathcal{N}_{m}^{\mathcal{S}}\right)_{m \in \mathbb{N}}$ is an arithmetico-geometric one, with $\mathcal{N}_{1}^{\mathcal{S}}=4$ as first term:

$$
\forall m \in \mathbb{N}: \quad \mathcal{N}_{m+1}^{\mathcal{S}}=4\left(\mathcal{N}_{m}^{\mathcal{S}}-1\right)-\left(\mathcal{N}_{m}^{\mathcal{S}}-1\right)=3 \mathcal{N}_{m}^{\mathcal{S}}-2 .
$$




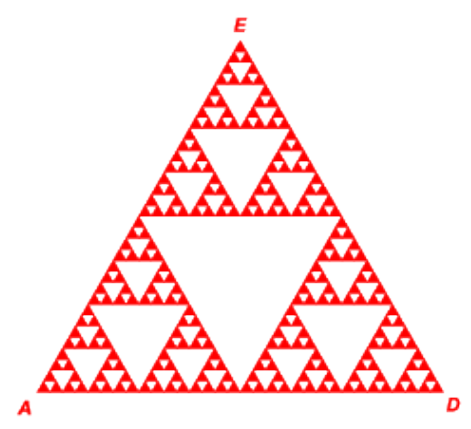

Figure 2.2. The points $A, D$ and $E$, as frontier vertices of the Sierpiński gasket

This leads to:

$$
\forall m \in \mathbb{N}^{\star}: \quad \mathcal{N}_{m+1}^{\mathcal{S}}=3^{m}\left(\mathcal{N}_{1}^{\mathcal{S}}-1\right)+1=3^{m+1}+1 .
$$

Definition 2.14 (Consecutive vertices on the graph $\mathcal{S} G^{\mathcal{C}}$ ). Two points $X$ and $Y$ of $\mathcal{S} G^{\mathcal{C}}$ will be called consecutive vertices of the graph $\mathcal{S} G^{\mathcal{C}}$ if there exists a natural integer $m$, and an integer $j$ of $\left\{1, \ldots, \mathcal{N}_{m}^{\mathcal{S}}-1\right\}$, such that:

$$
X=X_{j}^{m} \quad \text { and } \quad Y=X_{j+1}^{m}
$$

or:

$$
Y=X_{j}^{m} \quad \text { and } \quad X=X_{j+1}^{m} .
$$

Definition 2.15. For any positive integer $m$, the $\mathcal{N}_{m}^{\mathcal{S}}$ consecutive vertices of the graph $\mathcal{S} G_{m}^{\mathcal{C}}$ are also the vertices of $3^{m-1}$ trapezes $\mathcal{T}_{m, j}, 1 \leqslant j \leqslant 3^{m-1}$. For any integer $j$ such that $1 \leqslant j \leqslant 3^{m-1}$, one obtains each trapeze by linking the point number $j$ to the point number $j+1$ if $j \equiv i[4], 0 \leqslant i \leqslant 2$, and the point number $j$ to the point number $j-3$ if $j \equiv-1$ [4].

One has to consider those polygons as semi-closed ones, since, for any of those 4-gons, the starting vertex, i.e. the point number $j$, is not connected, on the graph $\mathcal{S} G_{m}^{\mathcal{C}}$, to the extreme one, i.e. the point number $j-3$, if $j \equiv-1[4]$. These trapezes generate a Borel set of $\mathbb{R}^{2}$.

In the sequel, we will denote by $\mathcal{T}_{1}$ the initial trapeze, the vertices of which are, respectively: $A, B, C, D$.

Definition 2.16 (Trapezoidal domain delimited by the graph $\mathcal{S} G_{m}^{\mathcal{C}}, m \in \mathbb{N}$ ). For any natural integer $m$, well call trapezoidal domain delimited by the graph $\mathcal{S} G_{m}^{\mathcal{C}}$, and denote by $\mathcal{D}\left(\mathcal{S} G_{m}^{\mathcal{C}}\right)$, the reunion of the $3^{m-1}$ trapezes $\mathcal{T}_{m, j}, 1 \leqslant j \leqslant 3^{m-1}$. 


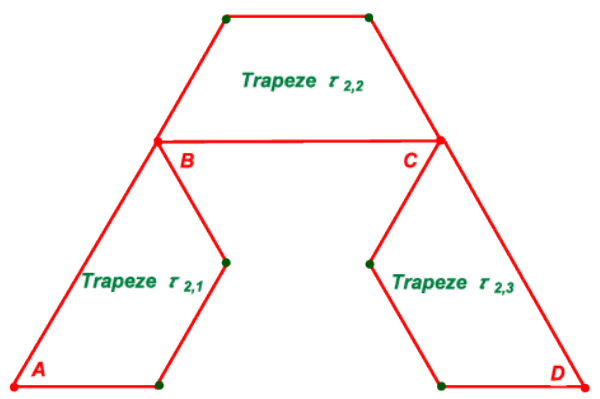

Figure 2.3. The trapezes $\mathcal{T}_{2,1}, \mathcal{T}_{2,2}$ and $\mathcal{T}_{2,3}$

Remark 2.17. The trapezoids $\mathcal{T}_{m, j}, 1 \leqslant j \leqslant 3^{m-1}$, are required in order to define a two-dimensional measure, as it will be exposed in the sequel.

Property 2.18. In the sequel, we set, for the Lebesgue measure of the first trapeze $\mathcal{T}_{1}$ :

$$
\mathcal{A}_{1}=\mathcal{A}\left(\mathcal{T}_{1}\right)=1
$$

One may note that this trapeze can be divided into three equilateral triangles, each of measure $\frac{1}{3}$, as it is shown in Figures 2.4 and 2.5.

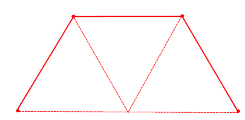

Figure 2.4. The initial trapeze, divided into three equilateral triangles.

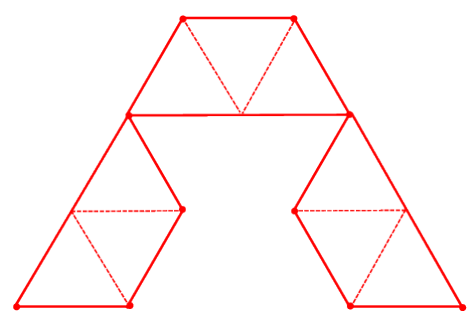

Figure 2.5. The trapezes, at step 2, each divided into three equilateral triangles.

By considering the prefractal graphs that approximate the Sierpinski gasket, as in [21] (see Figures 2.6 and 2.7), one can see that, at a given level $m \in \mathbb{N}^{\star}$, if each of the $3^{m}$ cells, which also corresponds to an equilateral triangle at level $m$ of the trapezes that approximate the Arrowhead Curve, 


\begin{tabular}{|c|c|c|}
\hline $\begin{array}{c}\text { Level of } \\
\text { approximation }\end{array}$ & Sierpiński gasket & Arrowhead Curve \\
\hline$m$ & $\begin{array}{c}\text { triangular cell, } \\
\text { measure } \frac{1}{3^{m}}\end{array}$ & $\begin{array}{c}\text { trapezoidal cell, } \\
\text { measure } \frac{1}{3^{m-1}}\end{array}$ \\
\hline
\end{tabular}

TABle 2.1. Comparison between the Sierpiński gasket and the Arrowhead Curve

has measure $\frac{1}{3^{m}}$, then, for any natural integer $m \geqslant 2$, the measure of a trapeze $\mathcal{T}_{m, j}, 1 \leqslant j \leqslant 3^{m-1}$ is given by:

$$
\mathcal{A}_{m}=\mathcal{A}\left(\mathcal{T}_{m, j}\right)=\frac{1}{3^{m-1}} .
$$

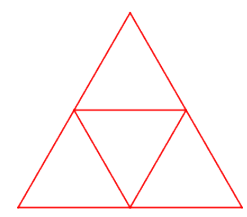

Figure 2.6. The gasket, at level 1

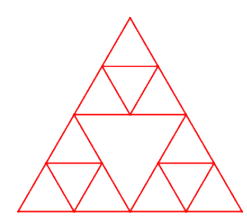

FigurE 2.7. The gasket, at level 2

This leads to the following correspondence, which, for the sake of clarity, is given in Table 2.1.

Definition 2.19. We will call trapezoidal domain delimited by the graph $\mathcal{S} G^{\mathcal{C}}$, and denote by $\mathcal{D}\left(\mathcal{S} G^{\mathcal{C}}\right)$, the limit:

$$
\mathcal{D}\left(\mathcal{S} G^{\mathcal{C}}\right)=\lim _{m \rightarrow+\infty} \mathcal{D}\left(\mathcal{S} G_{m}^{\mathcal{C}}\right)
$$

in the sense that, for any continuous function $u$ on the graph, and any measure with full support $\mu$ on $\mathbb{R}^{2}$ :

$$
\int_{\mathcal{D}\left(\mathcal{S} G^{\mathcal{C}}\right)} u d \mu=\lim _{m \rightarrow+\infty} \sum_{j=1}^{3^{m-1}} \sum_{X \text { is a vertex of } \mathcal{T}_{m, j}} \frac{u(X) p(X) \mu\left(\mathcal{T}_{m, j}\right)}{4}
$$

where: 
- $p(X)=1$ if $X$ is not a junction point between two consecutive trapezes;

- $p(X)=\frac{1}{2}$ if $X$ is the junction point between two consecutive trapezes $\mathcal{P}_{m, j}$ and $\mathcal{P}_{m, j+1}$.

Notation 2.20. In the sequel, we will denote by $d_{\mathbb{R}^{2}}$ the Euclidean distance on $\mathbb{R}^{2}$.

Definition 2.21 (Edge relation, on the graph $\mathcal{S} G^{\mathcal{C}}$ ). Given a natural integer $m$, two points $X$ and $Y$ of $\mathcal{S} G_{m}^{\mathcal{C}}$ will be called adjacent if and only if $X$ and $Y$ are two consecutive vertices of $\mathcal{S} G_{m}^{\mathcal{C}}$. We will write:

$$
X \underset{m}{\sim} Y \text {. }
$$

Given two points $X$ and $Y$ of the graph $\mathcal{S} G^{\mathcal{C}}$, we will say that $X$ and $Y$ are adjacent if and only if there exists a natural integer $m$ such that:

$$
X \underset{m}{\sim} Y .
$$

Property 2.22 (Euclidean distance of two adjacent vertices of $\mathcal{S} G_{m}^{\mathcal{C}}, m \in \mathbb{N}$ ). Given a natural integer $m$, and two points $X$ and $Y$ of $\mathcal{S} G_{m}^{\mathcal{C}}$ such that $X \underset{m}{\sim} Y:$

$$
d_{\mathbb{R}^{2}}(X, Y)=\frac{1}{2^{m}} .
$$

Property 2.23. The union $\bigcup_{m \in \mathbb{N}} V_{m}$ is dense in $\mathcal{S} G^{\mathcal{C}}$.

3. The Laplacian, By means of a natural approach

Definition 3.1 (Laplacian of order $m \in \mathbb{N}^{\star}$ ). For any strictly positive integer $m$, and any real-valued function $u$, defined on the set $V_{m}$ of the vertices of the graph $S G_{m}^{\mathcal{C}}$, we introduce the Laplacian of order $m, \Delta_{m}(u)$, by:

$$
\Delta_{m} u(X)=\sum_{Y \in V_{m}, Y_{\tilde{m}} X} c_{m} \frac{u(Y)-u(X)}{\ell_{m}^{2}}, \quad \forall X \in V_{m} \backslash V_{0},
$$

where $\ell_{m}=\frac{1}{2^{m}}$ and $c_{m}$ is a strictly positive constant.

Remark 3.2 (Determination of the constant $c_{m}, m \in \mathbb{N}$ ). For any natural integer $m$, the elementary length is:

$$
\ell_{m}=\frac{1}{2^{m}} .
$$

Since: $\# V_{m}=3^{m+1}+1$, the total length is thus:

$$
\left(3^{m+1}+1\right) \ell_{m}=\left(3^{m+1}+1\right) \times \frac{1}{2^{m}} \gg 1 .
$$


This amounts to place $K_{m}=3^{m+1}+1$ points on a circle, of perimeter:

$$
\frac{3^{m+1}+1}{2^{m}} \text {. }
$$

For $m \in \mathbb{N}$, the set of points on the circle is of the form:

$$
X_{k, m}=\frac{2 \pi k}{K_{m}}, \quad 0 \leqslant k \leqslant K_{m}-1 .
$$

Given a continuous function $u$ defined on $V_{m}$, we set for $k \in\left\{0, \ldots, K_{m}-1\right\}$ :

$$
U\left(X_{k, m}\right)=\sum_{j=0}^{K_{m}-1} u\left(X_{j, m}\right) e^{i j k \frac{2 \pi}{K_{m}}}
$$

Thus:

$$
\begin{aligned}
& U\left(X_{k, m}+\ell_{m}\right)=U\left(X_{k+1, m}\right)=\sum_{j=0}^{K_{m}-1} u\left(X_{j, m}\right) e^{i j(k+1) \frac{2 \pi}{K_{m}}}, \\
& U\left(X_{k, m}-\ell_{m}\right)=U\left(X_{k-1, m}\right)=\sum_{j=0}^{K_{m}-1} u\left(X_{j, m}\right) e^{i j(k-1) \frac{2 \pi}{K_{m}}}
\end{aligned}
$$

and:

$$
\begin{aligned}
& U\left(X_{k, m}+\ell_{m}\right)+U\left(X_{k, m}-\ell_{m}\right)-2 U\left(X_{k, m}\right)= \\
& =\sum_{j=0}^{K_{m}-1}\left\{e^{i j \frac{2 \pi}{K_{m}}}+e^{-i j \frac{2 \pi}{K_{m}}}-2\right\} u\left(X_{j, m}\right) e^{i j k \frac{2 \pi}{K_{m}}} .
\end{aligned}
$$

For $k \in\left\{0, \ldots, K_{m}-1\right\}$ :

$$
\frac{e^{i j \frac{2 \pi}{K_{m}}}+e^{-i j \frac{2 \pi}{K_{m}}}-2}{\ell_{m}^{2}}=\frac{2\left\{\cos \left(\frac{2 \pi j}{K_{m}}\right)-1\right\}}{\ell_{m}^{2}} .
$$

The spectrum of the normalized discrete Laplacian is thus:

$$
\begin{aligned}
\left\{\Lambda_{j}\left(h_{m}\right) \mid j=0, \ldots, K_{m}-1\right\} & = \\
& =c_{m}\left\{\frac{2\left\{\cos \left(\frac{2 \pi j}{K_{m}}\right)-1\right\}}{h_{m}^{2}} \mid j=0, \ldots, K_{m}-1\right\} .
\end{aligned}
$$

For $j$ in $\left\{0, \ldots, K_{m}-1\right\}$, when $m \rightarrow+\infty$ :

$$
c_{m} \frac{2\left\{\cos \left(\frac{2 \pi j}{K_{m}}\right)-1\right\}}{h_{m}^{2}} \sim c_{m} \frac{4 \pi^{2} j^{2} K_{m}^{-2}}{\ell_{m}^{2}} .
$$

The existence of the limit of the spectrum, when the integer $m$ tends towards infinity, requires:

$$
c_{m}=\ell_{m}^{2} K_{m}^{2}=4^{-m} 3^{2 m+2},
$$


i.e.

$$
\frac{c_{m}}{\ell_{m}^{2}}=K_{m}^{2} \sim 3^{2 m}=2^{2 m \delta}
$$

which corresponds to the value:

$$
\delta=\frac{\ln 3}{\ln 2} .
$$

One thus retrieves, as it could have been expected, the value of the Hausdorff dimension of the Sierpiński gasket:

$$
\delta=D_{\mathcal{S} G^{\mathcal{C}}}=D_{\mathcal{S} G}=\frac{\ln 3}{\ln 2} .
$$

\section{The Laplacian, By means of the Kigami and Strichartz APPROACH}

\subsection{Dirichlet forms.}

Definition 4.2. ([1, 5]) Given a measured space $(E, \mu)$, a Dirichlet form on $E$ is a bilinear symmetric form, that we will denote by $\mathcal{E}$, defined on a vectorial subspace $D$ dense in $L^{2}(E, \mu)$, such that:

(1) For any real-valued function $u$ defined on $D: \mathcal{E}(u, u) \geqslant 0$.

(2) $D$, equipped with the inner product which, to any pair $(u, v)$ of $D \times D$, associates:

$$
(u, v)_{\mathcal{E}}=(u, v)_{L^{2}(E, \mu)}+\mathcal{E}(u, v)
$$

is a Hilbert space.

(3) For any real-valued function $u$ defined on $D$, if:

$$
u_{\star}=\min (\max (u, 0), 1) \in D,
$$

then $\mathcal{E}\left(u_{\star}, u_{\star}\right) \leqslant \mathcal{E}(u, u)$ (Markov property, or lack of memory property).

Definition 4.3. ([12]) Let us consider a finite set $V$. The usual inner product of a pair $(u, v)$ of functions defined on $V$ is given by:

$$
\langle u, v\rangle=\sum_{p \in V} u(p) v(p) .
$$

A Dirichlet form on $V$ is a symmetric bilinear form $\mathcal{E}$, such that:

(1) For any real valued function $u$ defined on $V: \mathcal{E}(u, u) \geqslant 0$.

(2) $\mathcal{E}(u, u)=0$ if and only if $u$ is constant on $V$.

(3) For any real-valued function $u$ defined on $V$, if:

$$
u_{\star}=\min (\max (u, 0), 1)
$$


i.e.

$$
\forall p \in V: \quad u_{\star}(p)= \begin{cases}1, & \text { if } u(p) \geqslant 1, \\ u(p), & \text { if } 0<u(p)<1, \\ 0, & \text { if } u(p) \leqslant 0,\end{cases}
$$

then $\mathcal{E}\left(u_{\star}, u_{\star}\right) \leqslant \mathcal{E}(u, u)$ (Markov property).

4.4. Energy forms. Before introducing energy forms on our curve, we would like to recall that, a priori, Dirichlet forms solely depend on the topology of the sequence of graphs that approximate our curve. One may thus rightly think that, in this case, geometry won't be taken into account. It is not so. We shall see, further, how it plays its part.

Proposition 4.5 (Harmonic extension of a function, on the graph of Sierpiński arrowhead curve - Ramification constant). For any integer $m>1$, if $u$ is a real-valued function defined on $V_{m-1}$, its harmonic extension, denoted by $\tilde{u}$, is obtained as the extension of $u$ to $V_{m}$ which minimizes the energy:

$$
\mathcal{E}_{\mathcal{S} G_{m}^{\mathcal{C}}}(\tilde{u}, \tilde{u})=\sum_{\substack{X \sim Y \\ m}}(\tilde{u}(X)-\tilde{u}(Y))^{2}
$$

The link between $\mathcal{E}_{\mathcal{S} G_{m}^{\mathcal{C}}}$ and $\mathcal{E}_{\mathcal{S} G_{m-1}^{\mathcal{C}}}$ is obtained through the introduction of two strictly positive constants $r_{m}$ and $r_{m+1}$ such that:

$$
r_{m} \sum_{\substack{X \sim Y \\ m}}(\tilde{u}(X)-\tilde{u}(Y))^{2}=r_{m-1} \sum_{X \underset{m-1}{\sim} Y}(u(X)-u(Y))^{2} .
$$

Let us set

and

$$
r=\frac{1}{r_{1}}
$$

$$
\mathcal{E}_{m}(u)=r_{m} \sum_{\substack{X \sim Y \\ m}}(\tilde{u}(X)-\tilde{u}(Y))^{2},
$$

so, by induction, one gets:

$$
r_{m}=r_{1}^{m}=r^{-m}
$$

If $v$ is a real-valued function, defined on $V_{m-1}$, of harmonic extension $\tilde{v}$, we will write:

$$
\mathcal{E}_{m}(u, v)=r^{-m} \sum_{\substack{X \sim Y \\ m}}(\tilde{u}(X)-\tilde{u}(Y))(\tilde{v}(X)-\tilde{v}(Y)) .
$$

The constant $r^{-1}$, which can be interpreted as a topological one, will be called ramification constant. For further precision on the construction and existence of harmonic extensions, we refer to [17]. 
Remark 4.6 (Determination of the ramification constant $r^{-1}$ ). Let us denote by $u$ a real-valued, continuous function defined on $V_{1}$, and by $\tilde{u}$ its harmonic extension to $V_{2}$. Let also $a, b, c$ and $d$ be the values of $u$ on the four consecutive vertices of $V_{1}$ (see the following figure):

$$
u(A)=a, \quad u(B)=b, \quad u(C)=c, \quad u(D)=d,
$$

and by

(i) $e$ and $f$ the values of $\tilde{u}$ on the two consecutive vertices $E$ and $F$ that are between $A$ and $B$ :

$$
\tilde{u}(E)=e, \quad \tilde{u}(F)=f ;
$$

(ii) $g$ and $h$ the values of $\tilde{u}$ on the two consecutive vertices $G$ and $H$ that are between $B$ and $C$ :

$$
\tilde{u}(G)=g, \quad \tilde{u}(H)=h ;
$$

(iii) $i$ and $j$ the values of $\tilde{u}$ on the two consecutive vertices $I$ and $J$ that are between $C$ and $D$ :

$$
\tilde{u}(I)=i, \quad \tilde{u}(J)=j .
$$

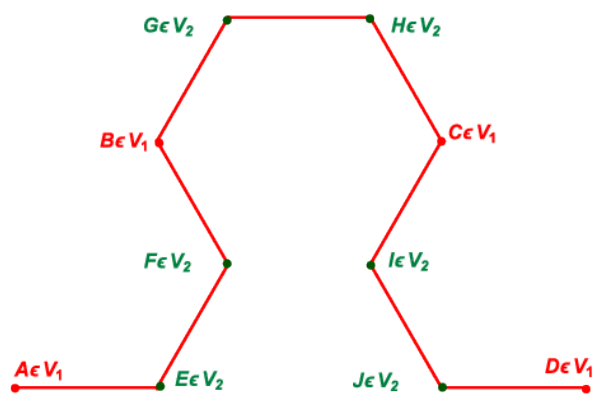

FiguRE 4.1. Determination of the ramification constant between graphs of level 1 and 2

One has:

$$
\begin{aligned}
\mathcal{E}_{\mathcal{S} G_{1}^{\mathcal{C}}}(u, u) & =(a-b)^{2}+(b-c)^{2}+(c-d)^{2}, \\
\mathcal{E}_{\mathcal{S} G_{2}^{\mathcal{C}}}(\tilde{u}, \tilde{u}) & =(a-e)^{2}+(e-f)^{2}+(b-f)^{2}+(g-b)^{2}+ \\
& \quad+(h-g)^{2}+(c-h)^{2}+(i-c)^{2}+(j-i)^{2}+(d-j)^{2} .
\end{aligned}
$$

Since the harmonic extension $\tilde{u}$ minimizes $\mathcal{E}_{\mathcal{S} G_{2}^{\mathcal{C}}}$, the values of $e, f, g, h, i$, $j$ are to be found among the critical points $e, f, g, h, i, j$ such that:

$$
\frac{\partial \mathcal{E}_{\mathcal{S} G_{2}^{\mathcal{C}}}(\tilde{u}, \tilde{u})}{\partial e}=0, \quad \frac{\partial \mathcal{E}_{\mathcal{S} G_{2}^{\mathcal{C}}}(\tilde{u}, \tilde{u})}{\partial f}=0, \quad \frac{\partial \mathcal{E}_{\mathcal{S} G_{2}^{\mathcal{C}}}(\tilde{u}, \tilde{u})}{\partial g}=0
$$




$$
\frac{\partial \mathcal{E}_{\mathcal{S} G_{2}^{\mathcal{C}}}(\tilde{u}, \tilde{u})}{\partial h}=0, \quad \frac{\partial \mathcal{E}_{\mathcal{S} G_{2}^{\mathcal{C}}}(\tilde{u}, \tilde{u})}{\partial i}=0, \quad \frac{\partial \mathcal{E}_{\mathcal{S} G_{2}^{\mathcal{C}}}(\tilde{u}, \tilde{u})}{\partial j}=0 .
$$

This leads to:

$$
\begin{aligned}
& e=\frac{2 a+b}{3}, \\
& f=\frac{2(a+2 b)}{3}, \\
& g=\frac{2 b+c}{3}, \\
& h=\frac{2(b+2 c)}{3}, \\
& i=\frac{2 c+d)}{3}, \\
& j=\frac{2(c+2 d)}{3},
\end{aligned}
$$

and:

Thus:

$$
\mathcal{E}_{\mathcal{S} G_{2}^{\mathcal{C}}}(\tilde{u}, \tilde{u})=\frac{1}{3} \mathcal{E}_{\mathcal{S} G_{1}^{\mathcal{C}}}(u, u) .
$$

$$
r^{-1}=3 .
$$

One may note that the ramification constant is exactly equal to one plus the number of points that arise in $V_{m+1}$, for any value of the strictly positive integer $m$, between two consecutive vertices of $V_{m}$.

Definition 4.7. By definition, the energy scaling factor is the strictly positive constant $\rho$ such that, for any integer $m>1$, and any real-valued function $u$ defined on $V_{m}$ :

$$
\mathcal{E}_{\mathcal{S} G_{m}^{\mathcal{C}}}(u, u)=\rho \mathcal{E}_{\mathcal{S} G_{m}^{\mathcal{C}}}\left(u_{\mid V_{m-1}}, u_{\mid V_{m-1}}\right)
$$

Proposition 4.8. The energy scaling factor $\rho$ is linked to the topology and the geometry of the fractal curve by means of the relation:

$$
\rho=r^{-1} \text {. }
$$

Definition 4.9. Let $m$ be a natural integer, and $u$ and $v$ two real valued functions, defined on the set

$$
V_{m}=\left\{X_{1}^{m}, \ldots, X_{\mathcal{N}_{m}^{s}}^{m}\right\}
$$

of the $\mathcal{N}_{m}^{\mathcal{S}}$ vertices of $\mathcal{S} G_{m}^{\mathcal{C}}$. We introduce the energy, on the graph $\mathcal{S} G_{m}^{\mathcal{C}}$, of the pair of functions $(u, v)$, as:

$$
\begin{aligned}
\mathcal{E}_{\mathcal{S} G_{m}^{\mathcal{C}}}(u, v) & =\sum_{i=1}^{\mathcal{N}_{m}^{\mathcal{S}}-1} r^{-m}\left(u\left(X_{i}^{m}\right)-u\left(X_{i+1}^{m}\right)\right)\left(v\left(X_{i}^{m}\right)-v\left(X_{i+1}^{m}\right)\right)= \\
& =\sum_{i=1}^{\mathcal{N}_{m}^{\mathcal{S}}-1} r^{-m}\left(u\left(X_{i}^{m}\right)-u\left(X_{i+1}^{m}\right)\right)\left(v\left(X_{i}^{m}\right)-v\left(X_{i+1}^{m}\right)\right) .
\end{aligned}
$$

For the sake of simplicity, we will write it under the form:

$$
\mathcal{E}_{\mathcal{S} G_{m}^{\mathcal{C}}}(u, v)=\sum_{\substack{X \sim Y \\ m}} r^{-m}(u(X)-u(Y))(v(X)-v(Y)) .
$$


Property 4.10. Given a natural integer $m$, the map, which, to any pair of real-valued, continuous functions $(u, v)$ defined on the set $V_{m}$ of the $\mathcal{N}_{m}$ vertices of $\mathcal{S} G_{m}^{\mathcal{C}}$, associates:

$$
\mathcal{E}_{\mathcal{S} G_{m}^{\mathcal{C}}}(u, v)=\sum_{X \sim{ }_{m}} r^{-m}(u(X)-u(Y))(v(X)-v(Y))
$$

is a Dirichlet form on $V_{m}$. Moreover:

$$
\mathcal{E}_{\mathcal{S} G_{m}^{\mathcal{C}}}(u, u)=0 \Longleftrightarrow u \text { is constant. }
$$

Definition 4.11 (Dirichlet form, for a pair of continuous functions defined on the graph $\mathcal{S} G^{\mathcal{C}}$ ). We define the Dirichlet form $\mathcal{E}$ which, to any pair of real-valued, continuous functions $(u, v)$ defined on the Sierpiński arrowhead curve $\mathcal{S} G^{\mathcal{C}}$, associates, subject to its existence:

$$
\begin{aligned}
\mathcal{E}(u, v) & =\lim _{m \rightarrow+\infty} \mathcal{E}_{m}\left(u_{\mid V_{m}}, v_{\mid V_{m}}\right)= \\
& \left.=\lim _{m \rightarrow+\infty} \sum_{\substack{\mathcal{m}_{m} Y \\
r^{-m}}} r_{\mid V_{m}}(X)-u_{\mid V_{m}}(Y)\right)\left(v_{\mid V_{m}}(X)-v_{\mid V_{m}}(Y)\right) .
\end{aligned}
$$

Definition 4.12 (Normalized energy, for a continuous function $u$, defined on the Sierpiński arrowhead curve). Taking into account that the sequence $\left(\mathcal{E}_{m}\left(u_{\mid V_{m}}\right)\right)_{m \in \mathbb{N}}$ is defined on

$$
V_{\star}=\bigcup_{i \in \mathbb{N}} V_{i}
$$

one defines the normalized energy, for a continuous function $u$, defined on the curve $\mathcal{S} G^{\mathcal{C}}$, by:

$$
\mathcal{E}(u)=\lim _{m \rightarrow+\infty} \mathcal{E}_{m}\left(u_{\mid V_{m}}\right)
$$

Notation 4.13. We will denote by $\operatorname{dom} \mathcal{E}$ the subspace of continuous functions defined on $\mathcal{S} G^{\mathcal{C}}$, such that: $\mathcal{E}(u)<+\infty$.

Notation 4.14. We will denote by $\operatorname{dom}_{0} \mathcal{E}$ the subspace of continuous functions defined on $\mathcal{S} G^{\mathcal{C}}$, which vanish on $V_{1}$, and such that: $\mathcal{E}(u)<+\infty$.

\subsection{Measures.}

Definition 4.16 (Self-similar measure, on the graph of the Sierpiński arrowhead curve). A measure $\mu$ on $\mathbb{R}^{2}$ will be said to be self-similar for the domain delimited by the Sierpiński arrowhead curve, if there exists a family of strictly positive pounds $\left(\mu_{1}, \mu_{2}, \mu_{3}\right)$ such that:

$$
\mu=\sum_{i=1}^{3} \mu_{i} \mu \circ \mathcal{H}_{i}^{-1}, \quad \quad \sum_{i=1}^{3} \mu_{i}=1 .
$$


For further precisions on self-similar measures, we refer to the works of J. E. Hutchinson (see [9]).

Property 4.17 (Building of a self-similar measure, for the domain delimited by the Sierpiński arrowhead curve). The Dirichlet forms mentioned in the above require a positive Radon measure with full support. The choice of a self-similar measure, which is, most of the time, built with regards to a reference set, of measure 1, appears, first, as very natural. R. S. Strichartz $[22,20]$, showed that one can simply consider auto-replicant measures $\tilde{\mu}$, i.e. measures $\tilde{\mu}$ such that:

$$
\tilde{\mu}=\sum_{i=1}^{3} \tilde{\mu}_{i} \tilde{\mu} \circ \mathcal{H}_{i}^{-1}
$$

where $\left(\tilde{\mu}_{1}, \tilde{\mu}_{2}, \tilde{\mu}_{3}\right)$ denotes a family of strictly positive pounds.

This latter approach appears as the best suited in our study, since, in the case of the graph $\mathcal{S} G^{\mathcal{C}}$, the initial set consists of the trapeze $\mathcal{T}_{0}$, the measure of which, equal to its surface, is not necessarily equal to 1 .

Let us assume that there exists a measure $\tilde{\mu}$ satisfying (4.1). Relation (4.1) yields, for any set of trapezes $\mathcal{T}_{m, j}, m \in \mathbb{N}$, and $1 \leqslant j \leqslant 3^{m-1}$ that

$$
\tilde{\mu}\left(\bigcup_{1 \leqslant j \leqslant 3^{m-1}} \mathcal{T}_{m, j}\right)=\sum_{i=1}^{3} \tilde{\mu}_{i} \tilde{\mu}\left(\mathcal{H}_{i}^{-1}\left(\bigcup_{1 \leqslant j \leqslant 3^{m-1}} \mathcal{T}_{m, j}\right)\right),
$$

and, in particular:

$$
\tilde{\mu}\left(\mathcal{H}_{1}\left(\mathcal{T}_{1}\right) \cup \mathcal{H}_{2}\left(\mathcal{T}_{1}\right) \cup \mathcal{H}_{3}\left(\mathcal{T}_{1}\right)\right)=\sum_{i=1}^{3} \tilde{\mu}_{i} \tilde{\mu}\left(\mathcal{T}_{1}\right),
$$

i.e.

$$
\sum_{i=1}^{3} \tilde{\mu}\left(\mathcal{H}_{i}\left(\mathcal{T}_{1}\right)\right)=\sum_{i=1}^{3} \tilde{\mu}_{i} \tilde{\mu}\left(\mathcal{T}_{1}\right)
$$

The convenient choice, for any $i$ of $\{1,2,3\}$, is:

$$
\tilde{\mu}_{i}=\frac{\tilde{\mu}\left(\mathcal{H}_{i}\left(\mathcal{T}_{1}\right)\right)}{\tilde{\mu}\left(\mathcal{T}_{1}\right)}=\frac{3}{4} .
$$

One can, from the measure $\tilde{\mu}$, build the self-similar measure $\mu$, such that:

$$
\mu=\sum_{i=1}^{3} \mu_{i} \mu \circ \mathcal{H}_{i}^{-1}
$$

where $\left(\mu_{i}\right)_{1 \leqslant i \leqslant 3}$ is a family of strictly positive pounds, the sum of which is equal to 1 . 
One has simply to set, for any $i$ of $\{1,2,3\}$ :

$$
\mu_{i}=\frac{4 \tilde{\mu}_{i}}{9} .
$$

The measure $\mu$ is self-similar, for the domain delimited by the Sierpiński arrowhead curve.

\subsection{Laplacian.}

Definition 4.19 (Topological Laplacian of order $m \in \mathbb{N}^{\star}$ ). For any strictly positive integer $m$, and any real-valued function $u$, defined on the set $V_{m}$ of the vertices of the graph $S G_{m}^{\mathcal{C}}$, we introduce the topological Laplacian of order $m, \Delta_{m}^{\tau}(u)$, by:

$$
\Delta_{m}^{\tau} u(X)=\sum_{Y \in V_{m}, Y \underset{m}{\sim} X}(u(Y)-u(X)), \forall X \in V_{m} \backslash V_{0} .
$$

Definition 4.20 (Harmonic function of order $m \in \mathbb{N}^{\star}$ ). Let $m$ be a strictly positive integer. A real-valued function $u$,defined on the set $V_{m}$ of the vertices of the graph $\mathcal{S} G_{m}^{\mathcal{C}}$, will be said to be harmonic of order $m$ if its Laplacian of order $m$ is null:

$$
\Delta_{m}^{\tau} u(X)=0 \quad \forall X \in V_{m} \backslash V_{0} .
$$

Definition 4.21 (Piecewise harmonic function of order $m \in \mathbb{N}^{\star}$ ). Given a strictly positive integer $m$, a real valued function $u$, defined on the set of vertices of $\mathcal{S} G^{\mathcal{C}}$, is said to be piecewise harmonic function of order $m$ if $u_{\mid V_{m}}$ is harmonic of order $m$.

Definition 4.22 (Existence domain of the Laplacian, for a continuous function on the graph $\mathcal{S} G^{\mathcal{C}}$ ). (see [1]). We will denote by dom $\Delta$ the existence domain of the Laplacian, on the graph $\mathcal{S} G^{\mathcal{C}}$, as the set of functions $u$ of $\operatorname{dom} \mathcal{E}$ such that there exists a continuous function on $\mathcal{S} G^{\mathcal{C}}$, denoted $\Delta u$, that we will call Laplacian of $u$, such that :

$$
\mathcal{E}(u, v)=-\int_{\mathcal{D}\left(\mathcal{S} G^{\mathcal{C}}\right)} v \Delta u d \mu \quad \text { for any } v \in \operatorname{dom}_{0} \mathcal{E} .
$$

Definition 4.23 (Harmonic function). A function $u$ belonging to $\operatorname{dom} \Delta$ will be said to be harmonic if its Laplacian is equal to zero.

Notation 4.24. In the following, we will denote by $\mathcal{H}_{0} \subset \operatorname{dom} \Delta$ the space of harmonic functions, i.e. the space of functions $u \in \operatorname{dom} \Delta$ such that:

$$
\Delta u=0 \text {. }
$$

Given a natural integer $m$, we will denote by $\mathcal{S}\left(\mathcal{H}_{0}, V_{m}\right)$ the space of spline functions "of level $m$ ", $u$, defined on $\mathcal{S} G^{\mathcal{C}}$, continuous, such that $u_{\mid V_{m}}$ is 
harmonic, i.e.:

$$
\Delta_{m}^{\tau}\left(u_{\mid V_{m}}\right)=0
$$

Property 4.25. For any natural integer $m$ :

$$
\mathcal{S}\left(\mathcal{H}_{0}, V_{m}\right) \subset \operatorname{dom} \mathcal{E} .
$$

Property 4.26. Let $m$ be a strictly positive integer, $X \notin V_{0}$ a vertex of the graph $\mathcal{S} G^{\mathcal{C}}$, and $\psi_{X}^{k} \in \mathcal{S}\left(\mathcal{H}_{0}, V_{k}\right)$ a spline function such that:

$$
\psi_{X}^{m}(Y)=\left\{\begin{array}{ll}
\delta_{X Y}, & \forall Y \in V_{k}, \\
0, & \forall Y \notin V_{m}
\end{array} \quad \text { where } \quad \delta_{X Y}= \begin{cases}1, & \text { if } X=Y, \\
0, & \text { otherwise }\end{cases}\right.
$$

Given a self-similar measure $\mu$ on the domain delimited by the Sierpiński arrowhead curve, one has:

$$
\begin{aligned}
\mathcal{E}\left(u, \psi_{X}^{m}\right) & =\mathcal{E}_{m}\left(u, \psi_{X}^{m}\right)= \\
& =\sum_{X \sim ⿱ ㇒} r^{-m}\left(u_{\mid V_{m}}(X)-u_{\mid V_{m}}(Y)\right)\left(\psi_{X \mid V_{m}}^{m}(X)-\psi_{X \mid V_{m}}^{m}(Y)\right)= \\
& =\sum_{X \sim \sim_{m} Y} r^{-m} \Delta_{m}^{\tau}(u)(X)\left(\psi_{X \mid V_{m}}^{m}(X)-\psi_{X \mid V_{m}}^{m}(Y)\right)= \\
& =-\int_{\mathcal{D}\left(\mathcal{S} G^{\mathcal{C}}\right)} \Delta^{\tau} u(X) \psi_{X}^{m}(X) d \mu \\
& \sim-\Delta u(X) \mathcal{A}_{m} .
\end{aligned}
$$

Thus

$$
\Delta u(X)=\lim _{m \rightarrow+\infty} \frac{1}{\mathcal{A}_{m}} r^{-m} \Delta_{m}^{\tau} u(X)=\lim _{m \rightarrow+\infty} 3^{2 m} \Delta_{m}^{\tau} u(X) .
$$

\section{The Mosco APPROACH}

Let us consider again the problem of energy forms on our curve, which cannot be obtained by means of a classical iterated function system, as it is the case with the Sierpiński gasket.

Such a problem was studied by U. Mosco [15], who specifically considered the case of what he called "the Sierpiński curve", or "Sierpiński string". Yet, he did not dealt with the curve itself, but with the Sierpiński gasket: "2D branches (...) meet together". Contrary to the arrowhead curve, the Sierpiński gasket exhibits self-similarity properties which turn it into a postcritically finite fractal (pcf fractal).

Yet, one can find interesting ideas in the work of U. Mosco. For instance, he suggests to generalize Riemannian models to fractals and relate the fractal analogous of gradient forms, i.e. the Dirichlet forms, to a metric 
that could reflect the fractal properties of the considered structure. The link is to be made by means of specific energy forms.

There are two major features that enable one to characterize fractal structures:

(i) their topology, i.e. their ramification;

(ii) their geometry.

The topology can be taken into account by means of classical energy forms (we refer to $[11,12,20,21]$ ). As for the geometry, again, things are not that simple to handle. U. Mosco introduces a strictly positive parameter, $\delta$, which is supposed to reflect the way ramification - or the iterative process that gives birth to the sequence of graphs that approximate the structure affects the initial geometry of the structure. For instance, if $m$ is a natural integer, $X$ and $Y$ two points of the initial graph $V_{1}$, and $\mathcal{M}$ a word of length $m$, the Euclidean distance $d_{\mathbb{R}^{2}}(X, Y)$ between $X$ and $Y$ is changed into the effective distance:

$$
\left(d_{\mathbb{R}^{2}}(X, Y)\right)^{\delta} .
$$

This parameter $\delta$ appears to be the one that can be obtained when building the effective resistance metric of a fractal structure (see [21]), which is obtained by means of energy forms. To avoid turning into circles, this means:

(i) either working, in a first time, with a value $\delta_{0}$ equal to one, and, then, adjusting it when building the effective resistance metric;

(ii) using existing results, as done in [3].

In the case of the Sierpinski gasket, at a step $m \in \mathbb{N}^{\star}$ of the iteration process, the effective distance between two adjacent points, as given in the work of U. Mosco, is

$$
\ell_{m}^{\delta_{\mathcal{S}} G}
$$

where

$$
\ell_{m}=\frac{1}{2^{m}} \quad \text { and } \quad \delta_{\mathcal{S}} G=\frac{\ln 3}{\ln 2}
$$

\section{Three APproaches, ONE LAPLACIAN}

Let us note that:

(i) The natural approach enables one to retrieve the usual Laplacian:

$$
\Delta u(X)=\lim _{m \rightarrow+\infty} \frac{c_{m}}{\ell_{m}^{2}} \Delta_{m}^{\tau} u(X)=\lim _{m \rightarrow+\infty} 3^{2 m} \Delta_{m}^{\tau} u(X)
$$

(ii) In the Kigami and Strichartz approach, in the case of the Sierpiński gasket $\mathcal{S} G$, the discrete Laplacians are graph ones (thus solely topological ones), which do not take account any metric. But, in fact, 
the geometry of the structure is indeed taken into account since, for a function $u$ in the domain of the Laplacian:

$$
\Delta u(X)=\lim _{m \rightarrow+\infty} r^{-m}\left(\int_{\mathcal{S} G} \psi_{X}^{m} d \mu\right)^{-1} \Delta_{m}^{\tau} u(X),
$$

where

$$
\int_{\mathcal{S} G} \psi_{X}^{m} d \mu=\frac{1}{3^{m}}
$$

is exactly the Lebesgue measure of the $m^{\text {th }}$-order triangular cell to which the vertex $X$ belongs.

If one denotes by $l_{m}>0$ a characteristic length of the $m^{\text {th }}$-order cell (the length of a side, for instance):

$$
\int_{\mathcal{S} G} \psi_{X}^{m} d \mu \sim l_{m}^{2}
$$

and thus retrieve a Riemannian expression, of the form:

$$
\Delta u(X)=\lim _{m \rightarrow+\infty} \frac{\text { Constant }(m)}{l_{m}^{2}} \Delta_{m}^{\tau} u(X) .
$$

(iii) In the case of the Mosco approach, topology and geometry are taken into account by means of a quasi-distance, built from the euclidean one $d_{\text {eucl }}$ between adjacent points $X$ and $Y$ such that $X \underset{m}{\sim} Y$ :

$$
d(X, Y)=\left(d_{\text {eucl }}(X, Y)\right)^{\delta}, \quad \delta>0 .
$$

The related energy writes:

$$
\mathcal{E}_{m}(u)=\sum_{X \sim \tilde{m}^{Y}} \frac{(\tilde{u}(X)-\tilde{u}(Y))^{2}}{d^{2}(X, Y)}
$$

This yields

$$
\Delta u(X)=\lim _{m \rightarrow+\infty} 3^{2 m} \Delta_{m}^{\tau} u(X)
$$

Satisfactorily, those three approaches generate the same Laplacian.

6.1. Spectrum of the Laplacian. In the following, $u$ denotes a continuous function on $\mathcal{S} G^{\mathcal{C}}$ which belongs to dom $\Delta$. We will apply the spectral decimation method developed by R. S. Strichartz [21], in the spirit of the works of M. Fukushima et T. Shima [4]. In order to determine the eigenvalues of the Laplacian $\Delta u$ built in the above, we concentrate first on the eigenvalues $\left(-\Lambda_{m}\right)_{m \in \mathbb{N}}$ of the sequence of graph Laplacians $\left(\Delta_{m} u\right)_{m \in \mathbb{N}}$, built on the discrete sequence of graphs $\left(\Gamma_{\mathcal{W}_{m}}\right)_{m \in \mathbb{N}}$. For any natural integer $m$, the restrictions of the eigenfunctions of the continuous Laplacian $\Delta u$ to 
the graph $\Gamma_{\mathcal{W}_{m}}$ are, also, eigenfunctions of the Laplacian $\Delta_{m}$, which leads to recurrence relations between the eigenvalues of order $m$ and $m+1$.

We thus aim at determining the solutions of the eigenvalue equation:

$$
-\Delta u=\Lambda u \quad \text { on } \mathcal{S} G^{\mathcal{C}}
$$

as limits, when the integer $m$ tends towards infinity, of the solutions of

$$
-\Delta_{m} u=\Lambda_{m} u \quad \text { on } V_{m} \backslash V_{0} .
$$

Let $m \geqslant 2$. We consider an eigenfunction $u_{m-1}$ on $V_{m-1} \backslash V_{1}$, for the eigenvalue $\Lambda_{m-1}$. The aim is to extend $u_{m-1}$ on $V_{m} \backslash V_{1}$ in a function $u_{m}$, which will itself be an eigenfunction of $\Delta_{m}$, for the eigenvalue $\Lambda_{m}$, and, thus, to obtain a recurrence relation between the eigenvalues $\Lambda_{m}$ and $\Lambda_{m-1}$. Given three consecutive vertices of $\mathcal{S} G_{m-1}^{\mathcal{C}}, X_{k}, X_{k+1}, X_{k+2}$, where $k$ denotes a generic natural integer, we will denote by $Y_{k+1}, Y_{k+2}$ the points of $V_{m} \backslash V_{m-1}$ such that: $Y_{k+1}, Y_{k+2}$ are between $X_{k}$ and $X_{k+1}$, by $Y_{k+4}, Y_{k+5}$, the points of $V_{m} \backslash V_{m-1}$ such that: $Y_{k+4}, Y_{k+5}$ are between $X_{k+1}$ and $X_{k+2}$, and by $Y_{k+7}, Y_{k+8}$, the points of $V_{m} \backslash V_{m-1}$ such that: $Y_{k+7}, Y_{k+8}$ are between $X_{k+2}$ and $X_{k+3}$. For the sake of consistency, let us set:

$$
Y_{k}=X_{k}, \quad Y_{k+3}=X_{k+1}, \quad Y_{k+6}=X_{k+2}, \quad Y_{k+9}=X_{k+3} .
$$

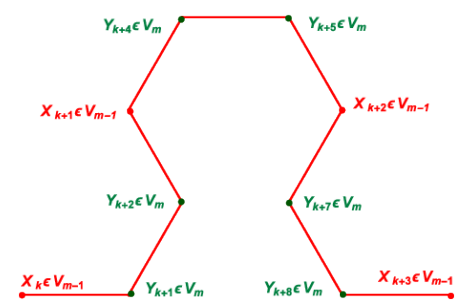

Figure 6.1 . The points $X_{k}, X_{k+1}, X_{k+2}, X_{k+3}$, and $Y_{k}, \ldots, Y_{k+9}$

The eigenvalue equation in $\Lambda_{m}$ leads to the following system:

$$
\left\{\begin{aligned}
\left\{\Lambda_{m}-2\right\} u_{m}\left(Y_{k+i+1}\right)=-u_{m}\left(Y_{k+i}\right) & -u_{m}\left(Y_{k+i+2}\right)= \\
& =-u_{m-1}\left(X_{k+i}\right)-u_{m}\left(Y_{k+i+2}\right) \\
\left\{\Lambda_{m}-2\right\} u_{m}\left(Y_{k+i+2}\right)=-u_{m}\left(Y_{k+i+1}\right) & -u_{m}\left(Y_{k+i+1}\right)= \\
& =-u_{m-1}\left(X_{k+i+1}\right)-u_{m}\left(Y_{k+i+1}\right)
\end{aligned}\right.
$$

where $0 \leqslant i \leqslant 2$.

The sequence $\left(u_{m}\left(Y_{k+i}\right)\right)_{0 \leqslant i \leqslant 9}$ satisfies a second order recurrence relation whose characteristic equation is

$$
r^{2}+\left\{\Lambda_{m}-2\right\} r+1=0
$$


Its discriminant is

$$
\delta_{m}=\left\{\Lambda_{m}-2\right\}^{2}-4=\omega_{m}^{2}, \quad \omega_{m} \in \mathbb{C},
$$

and the roots $r_{1, m}$ and $r_{2, m}$ of the characteristic equation are the scalar given by

$$
r_{1, m}=\frac{2-\Lambda_{m}-\omega_{m}}{2}, \quad r_{2, m}=\frac{2-\Lambda_{m}+\omega_{m}}{2} .
$$

One has then, for any natural integer $i$ of $\{0, \ldots, 9\}$

$$
u_{m}\left(Y_{k+i}\right)=\alpha_{m} r_{1, m}^{i}+\beta_{m} r_{2, m}^{i}
$$

where $\alpha_{m}$ and $\beta_{m}$ denote scalar constants.

The extension $u_{m}$ of $u_{m-1}$ to $V_{m} \backslash V_{1}$ has to be an eigenfunction of $\Delta_{m}$, for the eigenvalue $\Lambda_{m}$.

Since $u_{m-1}$ is an eigenfunction of $\Delta_{m-1}$, for the eigenvalue $\Lambda_{m-1}$, the sequence $\left(u_{m-1}\left(X_{k+i}\right)\right)_{0 \leqslant i \leqslant 9}$ must itself satisfy a second order linear recurrence relation which be the pendant, at order $m$, of the one satisfied by the sequence $\left(u_{m}\left(Y_{k+i}\right)\right)_{0 \leqslant i \leqslant 9}$ whose characteristic equation is:

$$
\left\{\Lambda_{m-1}-2\right\} r=-1-r^{2}
$$

and the discriminant

$$
\delta_{m-1}=\left\{\Lambda_{m-1}-2\right\}^{2}-4=\omega_{m-1}^{2}, \quad \omega_{m-1} \in \mathbb{C} .
$$

The roots $r_{1, m-1}$ and $r_{2, m-1}$ of this characteristic equation are given by

$$
r_{1, m-1}=\frac{2-\Lambda_{m-1}-\omega_{m-1}}{2}, \quad r_{2, m-1}=\frac{2-\Lambda_{m-1}+\omega_{m-1}}{2} .
$$

For any integer $i$ of $\{0, \ldots, 9\}$

$$
u_{m-1}\left(Y_{k+i}\right)=\alpha_{m-1} r_{1, m-1}^{i}+\beta_{m-1} r_{2, m-1}^{i},
$$

where $\alpha_{m-1}$ and $\beta_{m-1}$ denote scalar constants.

From this point, the compatibility conditions, imposed by spectral decimation, have to be satisfied:

$$
\begin{aligned}
u_{m}\left(Y_{k}\right) & =u_{m-1}\left(X_{k}\right), \\
u_{m}\left(Y_{k+3}\right) & =u_{m-1}\left(X_{k+1}\right), \\
u_{m}\left(Y_{k+6}\right) & =u_{m-1}\left(X_{k+2}\right), \\
u_{m}\left(Y_{k+9}\right) & =u_{m-1}\left(X_{k+3}\right),
\end{aligned}
$$

i.e.

$$
\begin{aligned}
\alpha_{m}+\beta_{m} & =\alpha_{m-1}+\beta_{m-1} & \mathcal{C}_{m} \\
\alpha_{m} r_{1, m}^{3}+\beta_{m} r_{2, m}^{3} & =\alpha_{m-1} r_{1, m-1}+\beta_{m-1} r_{2, m-1} & \mathcal{C}_{1, m}
\end{aligned}
$$




$$
\begin{array}{ll}
\alpha_{m} r_{1, m}^{6}+\beta_{m} r_{2, m}^{6}=\alpha_{m-1} r_{1, m-1}^{2}+\beta_{m-1} r_{2, m-1}^{2} & \mathcal{C}_{2, m} \\
\alpha_{m} r_{1, m}^{9}+\beta_{m} r_{2, m}^{9}=\alpha_{m-1} r_{1, m-1}^{3}+\beta_{m-1} r_{2, m-1}^{3} & \mathcal{C}_{3, m}
\end{array}
$$

where, for any natural integer $m, \alpha_{m}$ and $\beta_{m}$ are scalar constants (real or complex).

Since the graph $\mathcal{S} G_{m-1}^{\mathcal{C}}$ is linked to the graph $\mathcal{S} G_{m}^{\mathcal{C}}$ by a similar process to the one that links $\mathcal{S} G_{2}^{\mathcal{C}}$ to $\mathcal{S} G_{1}^{\mathcal{C}}$, one can legitimately consider that the constants $\alpha_{m}$ and $\beta_{m}$ do not depend on the integer $m$ :

$$
\forall m \in \mathbb{N}^{\star}: \quad \alpha_{m}=\alpha \in \mathbb{R}, \quad \beta_{m}=\beta \in \mathbb{R} .
$$

The above system writes:

$$
\begin{aligned}
& \alpha r_{1, m}^{3}+\beta r_{2, m}^{3}=\alpha r_{1, m-1}+\beta r_{2, m-1}, \\
& \alpha r_{1, m}^{6}+\beta r_{2, m}^{6}=\alpha r_{1, m-1}^{2}+\beta r_{2, m-1}^{2}, \\
& \alpha r_{1, m}^{8}+\beta r_{2, m}^{8}=\alpha r_{1, m-1}^{4}+\beta r_{2, m-1}^{4} .
\end{aligned}
$$

One has then to consider the following configurations:

(i) First case.

For any natural integer $m$

$$
r_{1, m} \in \mathbb{R}, \quad r_{2, m} \in \mathbb{R},
$$

and, more precisely:

$$
r_{1, m}<0, \quad r_{2, m}<0,
$$

since the function $\varphi$, which, to any real number $x \geqslant 4$, associates

$$
\varphi(x)=\frac{2-x+\varepsilon \sqrt{\{x-2\}^{2}-4}}{2}, \quad \varepsilon \in\{-1,1\},
$$

is strictly increasing on $(4,+\infty)$. Due to its continuity, is is a bijection of $(4,+\infty)$ on $\varphi((4,+\infty))=(-1,0)$.

Let us introduce the function $\phi$, which, to any real number $x \geqslant 2$, associates:

$$
\phi(x)=|\varphi(x)|=\frac{-2+x-\varepsilon \sqrt{\{x-2\}^{2}-4}}{2},
$$

where $\varepsilon \in\{-1,1\}$.

The function $\phi$ is a bijection of $(4,+\infty)$ onto $\phi((4,+\infty))=(0,1)$. We will denote by $\phi^{-1}$ its inverse bijection:

$$
\forall x \in(0,1): \quad \phi^{-1}(x)=\frac{(y+1)^{2}}{y} .
$$

One has then:

$$
\varphi\left(\Lambda_{m-1}\right)=\frac{2-\Lambda_{m-1}+\varepsilon \omega_{m-1}}{2} \leqslant 0 .
$$


This yields:

$$
(-1)^{3}\left(\varphi\left(\Lambda_{m}\right)\right)^{3}=\varphi\left(\Lambda_{m-1}\right) \leqslant 0,
$$

which leads to:

$$
\phi\left(\Lambda_{m}\right)=\left(\phi\left(\Lambda_{m-1}\right)\right)^{\frac{1}{3}}
$$

and

$$
\begin{aligned}
\Lambda_{m} & =\phi^{-1}\left(\left(\phi\left(\Lambda_{m-1}\right)\right)^{\frac{1}{3}}\right)=\frac{\left\{\left(\phi\left(\Lambda_{m-1}\right)\right)^{\frac{1}{3}}+1\right\}^{2}}{\left(\phi\left(\Lambda_{m-1}\right)\right)^{\frac{1}{3}}}= \\
& =\frac{\left\{\left(\frac{-2+\Lambda_{m-1}-\varepsilon \sqrt{\left\{\Lambda_{m-1}-2\right\}^{2}-4}}{2}\right)^{\frac{1}{3}}+1\right\}^{2}}{\left(\frac{-2+\Lambda_{m-1}-\varepsilon \sqrt{\left\{\Lambda_{m-1}-2\right\}^{2}-4}}{2}\right)^{\frac{1}{3}}} .
\end{aligned}
$$

(ii) Second case.

Notice that for any natural integer $m$

$$
r_{1, m} \in \mathbb{C} \backslash \mathbb{R}, \quad r_{2, m}=\overline{r_{1, m}} \in \mathbb{C} \backslash \mathbb{R}
$$

Let us introduce:

$$
\rho_{m}=\left|r_{1, m}\right| \in \mathbb{R}^{+}, \quad \theta_{m}=\arg r_{1, m} \text { if } r_{1, m} \neq 0 .
$$

The above system writes

$$
\left\{\begin{array}{l}
\rho_{m}^{3}\left\{\gamma \cos \left(3 \theta_{m}\right)+\delta \sin \left(3 \theta_{m}\right)\right\}=\rho_{m-1}\left\{\gamma \cos \left(\theta_{m-1}\right)+\delta \sin \left(\theta_{m-1}\right)\right\} \\
\rho_{m}^{6}\left\{\gamma \cos \left(6 \theta_{m}\right)+\delta \sin \left(6 \theta_{m}\right)\right\}=\rho_{m-1}^{2}\left\{\gamma \cos \left(2 \theta_{m-1}\right)+\delta \sin \left(2 \theta_{m-1}\right)\right\} \\
\rho_{m}^{9}\left\{\gamma \cos \left(9 \theta_{m}\right)+\delta \sin \left(9 \theta_{m}\right)\right\}=\rho_{m-1}^{3}\left\{\gamma \cos \left(3 \theta_{m-1}\right)+\delta \sin \left(3 \theta_{m-1}\right)\right\}
\end{array}\right.
$$

where $\gamma$ and $\delta$ denote real constants.

The system is satisfied if

$$
\left\{\begin{array}{l}
\rho_{m}^{3}=\rho_{m-1} \\
\theta_{m}=\frac{\theta_{m-1}}{3}
\end{array}\right.
$$

and thus

$$
\phi\left(\Lambda_{m}\right)=\left(\phi\left(\Lambda_{m-1}\right)\right)^{\frac{1}{N_{b}}},
$$

which leads to the same relation as in the previous case:

$$
\Lambda_{m}=\phi^{-1}\left(\left(\phi\left(\Lambda_{m-1}\right)\right)^{\frac{1}{3}}\right)=\frac{\left\{\left(\phi\left(\Lambda_{m-1}\right)\right)^{\frac{1}{3}}+1\right\}^{2}}{\left(\phi\left(\Lambda_{m-1}\right)\right)^{\frac{1}{3}}}=
$$




$$
=\frac{\left\{\left(\frac{-2+\Lambda_{m-1}-\varepsilon \sqrt{\left\{\Lambda_{m-1}-2\right\}^{2}-4}}{2}\right)^{\frac{1}{3}}+1\right\}^{2}}{\left(\frac{-2+\Lambda_{m-1}-\varepsilon \sqrt{\left\{\Lambda_{m-1}-2\right\}^{2}-4}}{2}\right)^{\frac{1}{3}}},
$$

where $\varepsilon \in\{-1,1\}$.

Proposition 6.2 (Comparison with the spectrum of the Sierpiński gasket). The eigenvalues $\left(\lambda_{m}^{\mathcal{S}} G\right)_{m \in \mathbb{N}}$ of the Laplacian on the Sierpinssi gasket are related by the quadratic equation

$$
\forall m \in \mathbb{N}^{\star}: \quad \lambda_{m-1}^{\mathcal{S}} G=\lambda_{m}^{\mathcal{S}} G\left(5-\lambda_{m}^{\mathcal{S}} G\right),
$$

(we refer to [20, 21] for further details). Thus, the respective spectrums of the Sierpinski gasket and arrowhead curve are distinct.

\section{Detalled study of the SPeCtrum of the Laplacian}

As exposed by R. S. Strichartz in [21], one may bear in mind that the eigenvalues can be grouped into two categories:

i) initial eigenvalues, which a priori belong to the set of forbidden values (as for instance $\Lambda=2$ );

ii) continued eigenvalues, obtained by means of spectral decimation.

We present, in the sequel, a detailed study of the spectrum of $\Delta$.

7.1. Eigenvalues and eigenvectors of $\Delta_{2}$. Let us recall that the vertices of the graph $\mathcal{S} G_{2}^{\mathcal{C}}$ are:

$$
X_{j}^{2}, \quad 1 \leqslant j \leqslant 10
$$

with

$$
X_{1}^{2}=A, \quad X_{4}^{2}=B, \quad X_{7}^{2}=C, \quad X_{10}^{2}=D .
$$

For the sake of simplicity, we will set here:

$$
X_{2}^{2}=E, \quad X_{3}^{2}=F, \quad X_{5}^{2}=G, \quad X_{6}^{2}=H, \quad X_{8}^{2}=I, \quad X_{9}^{2}=J .
$$

One may note that:

$$
\operatorname{Card}\left(V_{2} \backslash V_{1}\right)=10-4=6 .
$$

Let us denote by $u$ an eigenfunction for the eigenvalue $-\Lambda$ and put

$$
\begin{aligned}
& u(A)=a, \quad u(B)=b, \quad u(C)=c, \quad u(D)=d, \quad u(E)=e, \\
& u(F)=f, \quad u(G)=g, \quad u(H)=h, \quad u(I)=i, \quad u(J)=j .
\end{aligned}
$$




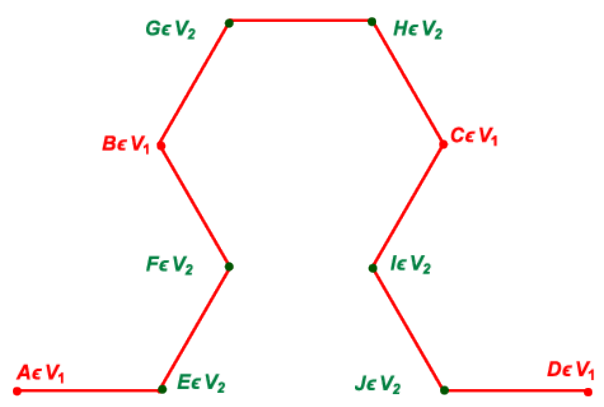

Figure 7.1. Successive values of an eigenfunction on $V_{2}$

One has then

$$
\left\{\begin{array}{l}
a+f=-(\Lambda-2) e \\
b+e=-(\Lambda-2) f \\
b+h=-(\Lambda-2) g \\
g+c=-(\Lambda-2) h \\
c+j=-(\Lambda-2) i \\
i+d=-(\Lambda-2) j
\end{array}\right.
$$

Notice that the only "Dirichlet eigenvalues", i.e. the ones related to the Dirichlet problem

$$
u_{\mid V_{1}}=0 \quad \text { i.e. } \quad u(A)=u(B)=u(C)=u(D)=0
$$

are obtained for:

$$
\left\{\begin{array} { l } 
{ f = - ( \Lambda - 2 ) e } \\
{ e = - ( \Lambda - 2 ) f } \\
{ h = - ( \Lambda - 2 ) g } \\
{ g = - ( \Lambda - 2 ) h } \\
{ j = - ( \Lambda - 2 ) i } \\
{ i = - ( \Lambda - 2 ) j }
\end{array} \quad \text { i.e. } \quad \left\{\begin{array}{l}
f=(\Lambda-2)^{2} f \\
e=(\Lambda-2)^{2} e \\
h=(\Lambda-2) h \\
g=(\Lambda-2)^{2} g \\
j=(\Lambda-2)^{2} i \\
i=(\Lambda-2)^{2} i
\end{array}\right.\right.
$$

The forbidden eigenvalue $\Lambda=2$ cannot thus be a Dirichlet one.

Consider the case where:

$$
(\Lambda-2)^{2}=1
$$

i.e.

$$
\Lambda=1 \quad \text { or } \quad \Lambda=3,
$$

which yields a three-dimensional eigenspace. The multiplicity of the eigenvalue $\Lambda=1$ is 3 . 
In the same way, the eigenvalue $\Lambda=3$ yields a three-dimensional eigenspace and its multiplicity is 3 .

Since the cardinal of $V_{2} \backslash V_{1}$ is:

$$
\mathcal{N}_{2}^{\mathcal{S}}-4=6
$$

one may note that we have the complete spectrum.

7.2. Eigenvalues of $\Delta_{m}, m \in \mathbb{N}, m \geqslant 3$. As before, one can easily check that the forbidden eigenvalue $\Lambda=2$ is not a Dirichlet one.

One can also check that $\Lambda_{m}=1$ and $\Lambda_{m}=3$ are eigenvalues of $\Delta_{m}$.

By induction, one may note that, due to the spectral decimation, the initial eigenvalue $\Lambda_{2}=1$ gives birth, at this $m^{\text {th }}$ step, to eigenvalues $\Lambda_{\hookrightarrow 1, m}$, and, in the same way, the initial eigenvalue $\Lambda_{2}=3$ gives birth, at this $m^{\text {th }}$ step, to eigenvalues $\Lambda_{\hookrightarrow 3, m}$.

The dimension of the Dirichlet eigenspace is equal to the cardinal of $V_{m} \backslash V_{1}$, i.e.

\[ \mathcal{N}_{m}^{\mathcal{S}}-\mathcal{N}_{1}^{\mathcal{S}}=3^{m}-3 \]
\begin{tabular}{|l|c|c|c|c|}
\hline Level & $m$ & 2 & 3 & 4 \\
\hline Cardinal of the Dirichlet spectrum & $3^{m}-3$ & 6 & 24 & 78 \\
\hline
\end{tabular}

\subsection{Eigenvalue counting function.}

Definition 7.4 (Eigenvalue counting function). Let us introduce the eigenvalue counting function, related to $\mathcal{S} G^{\mathcal{C}} \backslash V_{1}$, such that, for any positive number $x$

$$
\mathcal{N}^{\mathcal{S} G^{\mathcal{C}} \backslash V_{1}}(x)=\operatorname{Card}\{\Lambda \text { is a Dirichlet eigenvalue of }-\Delta: \Lambda \leqslant x\}
$$

Property 7.5. Given an integer $m \geqslant 2$, the cardinal of $V_{m} \backslash V_{1}$ is:

$$
\mathcal{N}_{m}^{\mathcal{S}}-\mathcal{N}_{1}^{\mathcal{S}}=3^{m}-3 \text {. }
$$

This leads to the existence of a strictly positive constant $C$ such that:

$$
\mathcal{N}^{\mathcal{S} G^{\mathcal{C}}}\left(C 9^{m}\right)=3^{m}-3 \text {. }
$$

If one looks for an asymptotic growth rate of the form

$$
\mathcal{N}^{\mathcal{S} G^{\mathcal{C}}}(x) \sim x^{\alpha_{\mathcal{S}} \mathcal{C}}
$$

then

$$
\alpha_{\mathcal{S} G^{\mathcal{C}}}=\frac{1}{2}
$$

which is not the same value as in the case of the Sierpiński gasket (we refer to $[21])$ :

$$
\alpha_{\mathcal{S} G^{\mathcal{C}}}<\alpha_{\mathcal{S} G}=\frac{\ln 3}{\ln 5}
$$


It turns out then that increasing the number of points, and the number of connections, increases the value of the Weyl exponent $\alpha$.

\section{Conclusion}

In an interesting way, it appears that if the Laplacian on the Sierpinski gasket and on the arrowhead curve are equivalent, they do not have the same spectral properties, especially as regards the exponent of the Weyl formula. In so far as different topologies are involved, it seems as a rather natural result. Yet, the geometry is the same. So what? We do not have the answer to this question, but one may argue that it comes from the non-uniqueness of the Laplacian. Our study has, also, put the light on the fact that increasing the number of points of a structure, and the number of connections, as it is the case in the triangle, increases the value of the Weyl exponent.

Thanks. To G. for the light put on the Laplacian, and for all that followed. The author would also like to thank the anonymous referee for his careful reading, and his very pertinent suggestions and advices, which helped a lot improving the original work.

\section{REFERENCES}

[1] A. Beurling, J. Deny. Espaces de Dirichlet. I. Le cas élémentaire. Acta Math., 99:203224, 1958, doi: 10.1007/BF02392426.

[2] K. J. Falconer. The geometry of fractal sets, volume 85 of Cambridge Tracts in Mathematics. Cambridge University Press, Cambridge, 1986.

[3] U. R. Freiberg, M. R. Lancia. Energy form on a closed fractal curve. Z. Anal. Anwendungen, 23(1):115-137, 2004, doi: 10.4171/ZAA/1190.

[4] M. Fukushima, T. Shima. On a spectral analysis for the Sierpiński gasket. Potential Anal., 1(1):1-35, 1992, doi: 10.1007/BF00249784.

[5] Masatoshi Fukushima, Yoichi Ōshima, Masayoshi Takeda. Dirichlet forms and symmetric Markov processes, volume 19 of De Gruyter Studies in Mathematics. Walter de Gruyter \& Co., Berlin, 1994, doi: 10.1515/9783110889741.

[6] J. Harrison. Stokes theorem for nonsmooth chains. Bull. of AMS, 29(2):235-242, 1993.

[7] Jenny Harrison, Alec Norton. Geometric integration on fractal curves in the plane. Indiana Univ. Math. J., 40(2):567-594, 1991, doi: 10.1512/iumj.1991.40.40027.

[8] Jenny Harrison, Alec Norton. The Gauss-Green theorem for fractal boundaries. Duke Math. J., 67(3):575-588, 1992, doi: 10.1215/S0012-7094-92-06724-X.

[9] John E. Hutchinson. Fractals and self-similarity. Indiana Univ. Math. J., 30(5):713747, 1981, doi: 10.1512/iumj.1981.30.30055.

[10] Boris A. Kats. The Stieltjes integral along fractal curve. Matematiche (Catania), 54(1):159-173 (2000), 1999.

[11] Jun Kigami. A harmonic calculus on the Sierpiński spaces. Japan J. Appl. Math., 6(2):259-290, 1989, doi: 10.1007/BF03167882.

[12] Jun Kigami. Harmonic calculus on p.c.f. self-similar sets. Trans. Amer. Math. Soc., 335(2):721-755, 1993, doi: 10.2307/2154402. 
[13] Jun Kigami. Harmonic analysis for resistance forms. J. Funct. Anal., 204(2):399-444, 2003, doi: 10.1016/S0022-1236(02)00149-0.

[14] Benoit B. Mandelbrot. The fractal geometry of nature. W. H. Freeman and Co., San Francisco, Calif., 1982. Schriftenreihe für den Referenten. [Series for the Referee].

[15] Umberto Mosco. Energy functionals on certain fractal structures. J. Convex Anal., 9(2):581-600, 2002. Special issue on optimization (Montpellier, 2000).

[16] Heinz-Otto Peitgen, Hartmut Jürgens, Dietmar Saupe. Chaos and fractals. SpringerVerlag, New York, second edition, 2004, doi: 10.1007/b97624. New frontiers of science, With a foreword by Mitchell J. Feigenbaum.

[17] C. Sabot. Existence and uniqueness of diffusions on finitely ramified self-similar fractals. Ann. Sci. École Norm. Sup. (4), 30(5):605-673, 1997, doi: 10.1016/S00129593(97)89934-X.

[18] Hans Sagan. Approximating Polygons for Lebesgue's and Schoenberg's Space Filling Curves. Amer. Math. Monthly, 93(5):361-368, 1986, doi: 10.2307/2323593.

[19] Hans Sagan. Space-filling curves. Universitext. Springer-Verlag, New York, 1994, doi: 10.1007/978-1-4612-0871-6.

[20] Robert S. Strichartz. Analysis on fractals. Notices Amer. Math. Soc., 46(10):1199-1208, 1999.

[21] Robert S. Strichartz. Differential equations on fractals. Princeton University Press, Princeton, NJ, 2006. A tutorial.

[22] Robert S. Strichartz, Arthur Taylor, Tong Zhang. Densities of self-similar measures on the line. Experiment. Math., 4(2):101-128, 1995, http://projecteuclid.org/euclid.em/ 1047931621.

[23] Hassler Whitney. Geometric integration theory. Princeton University Press, Princeton, N. J., 1957.

[24] W. Wunderlich. Über Peano-Kurven. Elem. Math., 28:1-10, 1973.

Received: October 3, 2019, accepted: May 4, 2020.

Claire David

Sorbonne Université - CNRS, UMR 7598, Laboratoire JaCques-Louis Lions, 4, Place Jussieu 75005, Paris, France

Email: Claire.David@sorbonne-universite.fr

ORCID: orcid.org/0000-0002-4729-0733 